\title{
SUPPORTING INFORMATION Stochastic Noise in Single-Nanoparticle Catalysis
}

\author{
Dinumol Devasia ${ }^{\dagger}$ and Prashant K. Jain ${ }^{\dagger \# *}$ \\ 'Department of Chemistry, ${ }^{\ddagger}$ Materials Research Laboratory, and ${ }^{\#}$ Beckman Institute for \\ Advanced Science and Technology, University of Illinois at Urbana-Champaign, Urbana, Illinois \\ 61801, United States
}

*Corresponding author E-mail: jain@illinois.edu 


\section{Supporting Tables}

Density functional theory (DFT)-computed Raman vibrational modes of species. Tables S1S9 below provide for each species the calculated vibrational frequencies, the corresponding dominant local mode/s, and their \% contribution to the frequency estimated by the potential energy distribution (PED) distribution. Positive \% contribution values indicate symmetric modes and negative values indicate antisymmetric modes of vibration. The following symbols are used to indicate various vibrational modes: $v_{\mathrm{s}}-$ symmetric stretching; $v_{\text {as }}$-antisymmetric stretching; $\delta-$ bending; $\rho$-rocking; $\tau$-twisting; $\omega$-wagging; $\phi$-torsion.

Table S1. DFT-computed Raman frequencies and normal mode analysis for 2,3-furandiol

\begin{tabular}{|c|c|}
\hline $\begin{array}{c}\text { Frequency, } \\
\mathrm{cm}^{-1}\end{array}$ & $\begin{array}{c}\text { Major vibrational } \\
\text { component (PED, \%) }\end{array}$ \\
\hline 827 & $\phi \mathrm{HCCC}(76)$ \\
\hline 905 & $\delta \mathrm{CCO}(68)$ \\
\hline 1048 & $v_{\mathrm{as}} \mathrm{OC}(-40)$ \\
\hline 1075 & $\begin{array}{l}\delta \text { HOC (-17); } \\
\delta \text { COC (22) }\end{array}$ \\
\hline 1153 & $v_{\mathrm{as}} \mathrm{OC}(-34)$ \\
\hline 1187 & $v_{\mathrm{s}} \mathrm{CC}(27)$ \\
\hline 1264 & $\delta \mathrm{HCO}(34)$ \\
\hline 1302 & $\delta$ HOC $(-29)$ \\
\hline 1360 & $v_{\mathrm{as}} \mathrm{CC}(-29)$ \\
\hline 1482 & $\delta \operatorname{CCO}(26)$ \\
\hline 1548 & $v_{\mathrm{s}} \mathrm{CC}(68)$ \\
\hline 1705 & $v_{\mathrm{s}} \mathrm{CC}(63)$ \\
\hline
\end{tabular}


Table S2. DFT-computed Raman frequencies and normal mode analysis for acetate

\begin{tabular}{|c|c|}
\hline $\begin{array}{c}\text { Frequency, } \\
\mathrm{cm}^{-1}\end{array}$ & $\begin{array}{c}\text { Major vibrational } \\
\text { component (PED, } \%)\end{array}$ \\
\hline 862 & $v_{\mathrm{s}} \mathrm{CC}(63)$ \\
\hline 994 & $\phi \mathrm{HCCO}(-66)$ \\
\hline 1029 & $\phi \mathrm{HCCO}(-53)$ \\
\hline 1319 & $\delta \mathrm{HCH}(85)$ \\
\hline 1345 & $v_{\mathrm{s}} \mathrm{OC}(78)$ \\
\hline 1461 & $\delta \mathrm{HCH}(-76)$ \\
\hline 1475 & $\delta \mathrm{HCH}(79)$ \\
\hline 1648 & $v_{\mathrm{s}} \mathrm{OC}(-93)$ \\
\hline
\end{tabular}

Table S3. DFT-computed Raman frequencies and normal mode analysis for pyruvate

\begin{tabular}{|c|c|}
\hline $\begin{array}{c}\text { Frequency, } \\
\mathrm{cm}^{-1}\end{array}$ & $\begin{array}{c}\text { Major vibrational } \\
\text { component (PED, } \%)\end{array}$ \\
\hline 1006 & $\phi$ HCCC $(55)$ \\
\hline 1153 & $\phi \mathrm{HCCC}(28)$ \\
\hline 1322 & $v_{\mathrm{s}}$ OC $(85)$ \\
\hline 1358 & $\delta \mathrm{HCH}(77)$ \\
\hline 1450 & $\delta \mathrm{HCH}(52)$ \\
\hline 1697 & $\nu_{\mathrm{s}} \mathrm{OC}(94)$ \\
\hline 1755 & $\nu_{\mathrm{s}} \mathrm{OC}(92)$ \\
\hline
\end{tabular}

Table S4. DFT-computed Raman frequencies and normal mode analysis for carbonate

\begin{tabular}{|c|c|}
\hline $\begin{array}{c}\text { Frequency, } \\
\mathrm{cm}^{-1}\end{array}$ & $\begin{array}{c}\text { Major vibrational } \\
\text { component }(\mathrm{PED}, \%)\end{array}$ \\
\hline 856 & $\phi$ OCCC $(100)$ \\
\hline 1010 & $v_{\mathrm{s}} \mathrm{OC}(100)$ \\
\hline 1318 & $v_{\mathrm{s}} \mathrm{OC}(-58)$ \\
\hline
\end{tabular}


Table S5. DFT-computed Raman frequencies and normal mode analysis for butene

\begin{tabular}{|c|c|}
\hline $\begin{array}{c}\text { Frequency, } \\
\mathrm{cm}^{-1}\end{array}$ & $\begin{array}{c}\text { Major vibrational } \\
\text { component }(\mathrm{PED}, \%)\end{array}$ \\
\hline 989 & $\delta$ HCC $(-56)$ \\
\hline 1019 & $v_{\mathrm{s} C C}(-53)$ \\
\hline 1030 & $\phi$ HCCC $(-62)$ \\
\hline 1093 & $\phi$ HCCC $(-22)$ \\
\hline 1198 & $\delta$ HCC $(-24)$ \\
\hline 1290 & $\delta$ HCC $(-41)$ \\
\hline 1319 & $\delta$ HCC $(63)$ \\
\hline 1344 & $\phi$ HCCC $(-60)$ \\
\hline 1408 & $\delta$ HCH $(95)$ \\
\hline 1453 & $\delta$ HCH $(73)$ \\
\hline 1483 & $\delta$ HCH $(-80)$ \\
\hline 1498 & $\delta$ HCH $(-72)$ \\
\hline 1506 & $\delta$ HCH $(-73)$ \\
\hline 1699 & $v_{s}$ CC $(70)$ \\
\hline
\end{tabular}

Table S6. DFT-computed Raman frequencies and normal mode analysis for glyceraldehyde

\begin{tabular}{|c|c|}
\hline $\begin{array}{c}\text { Frequency, } \\
\mathrm{cm}^{-1}\end{array}$ & $\begin{array}{c}\text { Major vibrational } \\
\text { component (PED, \%) }\end{array}$ \\
\hline 907 & $\phi$ HCCC $(-40)$ \\
\hline 1035 & $v_{\mathrm{s}} \mathrm{CC}(32)$ \\
\hline 1072 & $v_{\mathrm{s}} \mathrm{OC}(70)$ \\
\hline 1086 & $v_{\mathrm{s}} \mathrm{OC}(49)$ \\
\hline 1109 & $v_{\mathrm{s}} \mathrm{OC}(15)$ \\
\hline 1199 & $\phi \mathrm{HCCC}(-39)$ \\
\hline 1219 & $\delta \mathrm{HOC}(-28)$ \\
\hline 1310 & $\delta \mathrm{HCO}(41)$ \\
\hline 1365 & $\delta \mathrm{HCO}(67)$ \\
\hline 1382 & $\delta \mathrm{HCO}(39)$ \\
\hline 1407 & $\delta \mathrm{HOC}(29)$ \\
\hline 1439 & $\delta \mathrm{HCO}(-29)$ \\
\hline 1506 & $\delta \mathrm{HCH}(91)$ \\
\hline 1793 & $v_{\mathrm{s}} \mathrm{OC}(92)$ \\
\hline
\end{tabular}


Table S7. DFT-computed Raman frequencies and normal mode analysis for methylglyoxal

\begin{tabular}{|c|c|}
\hline $\begin{array}{c}\text { Frequency, } \\
\mathrm{cm}^{-1}\end{array}$ & $\begin{array}{c}\text { Major vibrational } \\
\text { component (PED, \%) }\end{array}$ \\
\hline 901 & $\phi$ HCCO $(-64)$ \\
\hline 1009 & $\phi$ HCCC $(-46)$ \\
\hline 1072 & $\phi$ HCCC $(22)$ \\
\hline 1238 & $v_{\text {as }}$ CC $(-47)$ \\
\hline 1355 & $\delta$ HCO $(92)$ \\
\hline 1392 & $\delta$ HCH $(45)$ \\
\hline 1460 & $\delta$ HCH $(-41)$ \\
\hline 1784 & $\nu_{\mathrm{s}}$ OC $(90)$ \\
\hline 1801 & $\nu_{s} \mathrm{OC}(95)$ \\
\hline
\end{tabular}

Table S8. DFT-computed Raman frequencies and normal mode analysis for oxalate

\begin{tabular}{|c|c|}
\hline $\begin{array}{c}\text { Frequency, } \\
\mathrm{cm}^{-1}\end{array}$ & $\begin{array}{c}\text { Major vibrational } \\
\text { component (PED, \%) }\end{array}$ \\
\hline 861 & $\phi_{\text {out }}$ OCOC (100) \\
\hline 1289 & $v_{\mathrm{s}}$ OC $(93)$ \\
\hline 1336 & $v_{\mathrm{s}}$ OC $(87)$ \\
\hline 1577 & $v_{\mathrm{s}}$ OC $(88)$ \\
\hline 1605 & $v_{\mathrm{s}}$ OC $(98)$ \\
\hline
\end{tabular}

Table S9. DFT-computed Raman frequencies and normal mode analysis for formate

\begin{tabular}{|c|c|}
\hline $\begin{array}{c}\text { Frequency, } \\
\mathrm{cm}^{-1}\end{array}$ & $\begin{array}{c}\text { Major vibrational } \\
\text { component (PED, \%) }\end{array}$ \\
\hline 1042 & $\phi_{\text {out }}$ COOH (100) \\
\hline 1336 & $v_{\mathrm{s}}$ OC $(89)$ \\
\hline 1375 & $\delta$ HCO $(94)$ \\
\hline 1660 & $v_{\mathrm{s}} \mathrm{OC}(94)$ \\
\hline 2563 & $v_{\mathrm{s}} \mathrm{CH}(99)$ \\
\hline
\end{tabular}




\section{Supporting Figures}

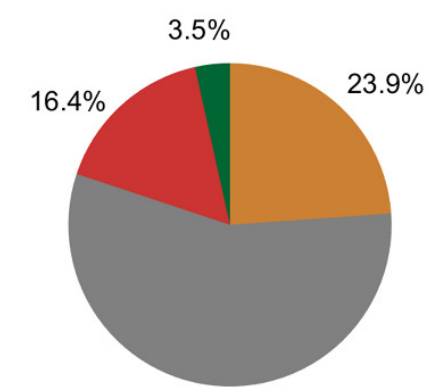

$56.2 \%$

NP scatterer 7

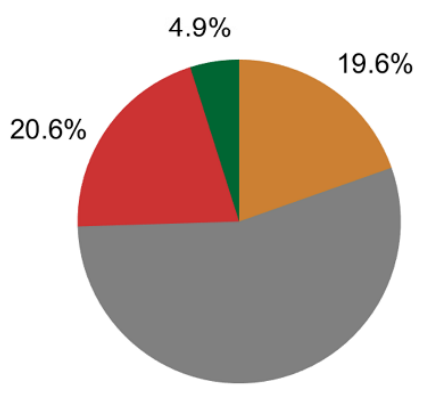

$54.9 \%$

NP scatterer 10

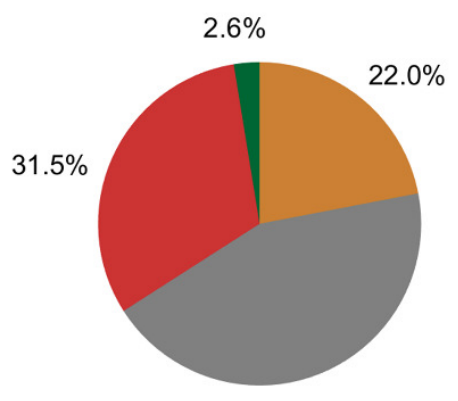

$44.0 \%$

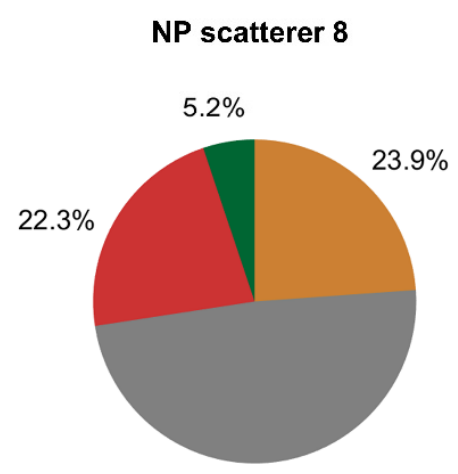

$48.7 \%$

NP scatterer 11

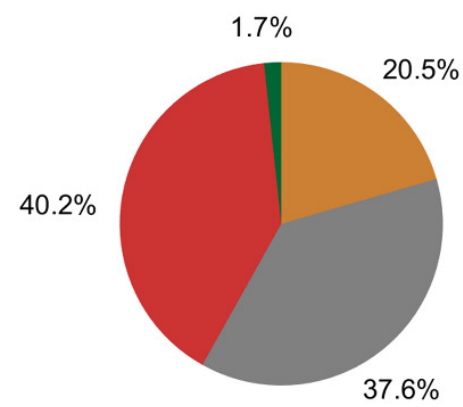

NP scatterer 9

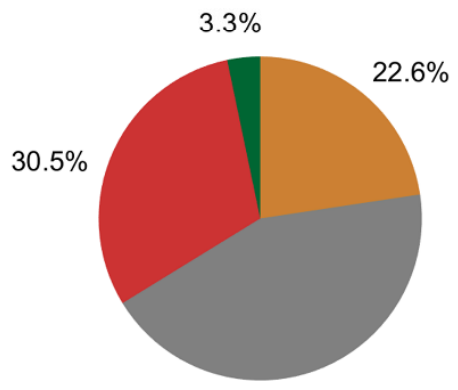

$43.6 \%$

NP scatterer 12

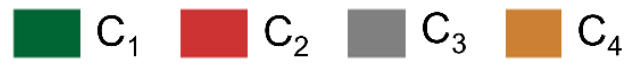

Figure S1. Variation in $\mathrm{CO}_{2} \mathrm{RR}$ selectivity from one nanoscale location to another. Additional examples of pie charts showing for individual $\mathrm{Ag}$ NP scatterers the selectivities (\%) of $\mathrm{C}_{1}-\mathrm{C}_{4}$ formation in $\mathrm{CO}_{2} \mathrm{RR}$ under plasmonic excitation over the full time-course of observation. The pie chart is based on 201, 273, 117, 102, 310, and 548 active assigned spectra respectively for NP scatterer 7, NP scatterer 8, NP scatterer 9, NP scatterer 10, NP scatterer 11, and NP scatterer 12. 


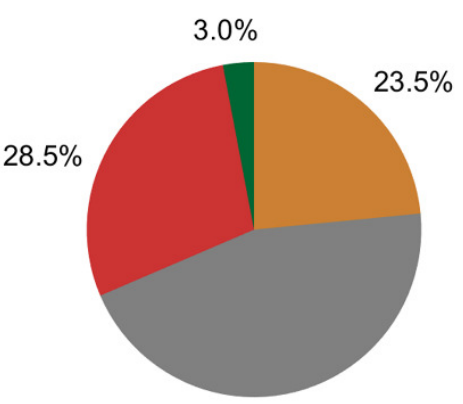

$45.1 \%$

Time segment 1 ( $N=1304)$

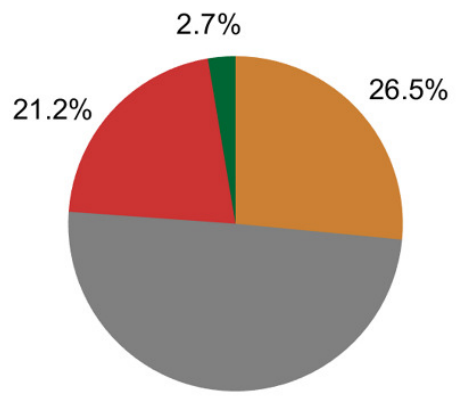

$49.6 \%$

Time segment $4(\mathrm{~N}=679)$

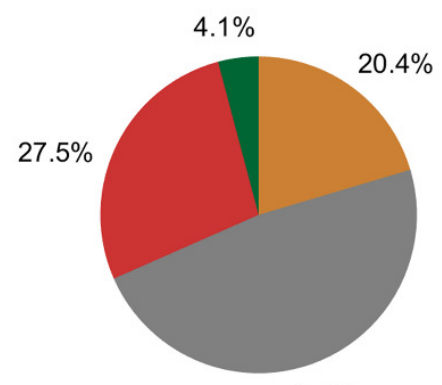

$48.0 \%$

Time segment 7 ( $N=750)$

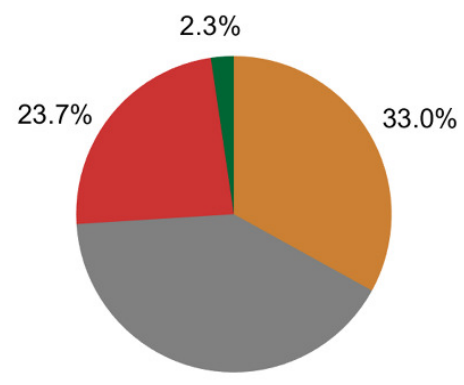

$41.0 \%$

Time segment $10(\mathrm{~N}=427)$

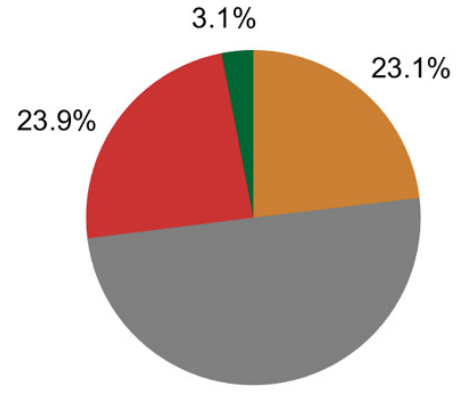

$49.9 \%$

Time segment 2 ( $N=1112)$

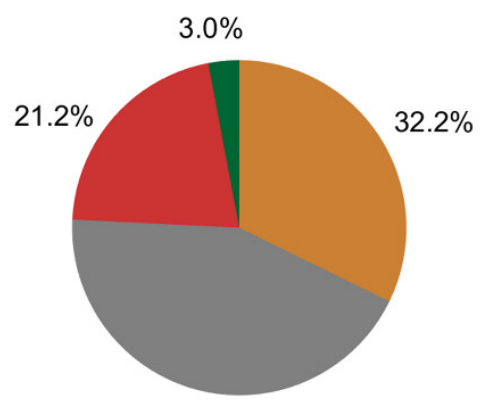

$43.5 \%$

Time segment 5 ( $N=673)$

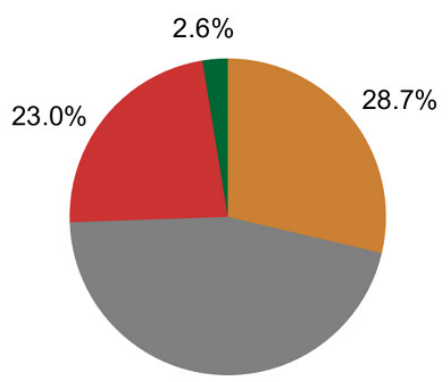

$45.8 \%$

Time segment $8(\mathrm{~N}=544)$

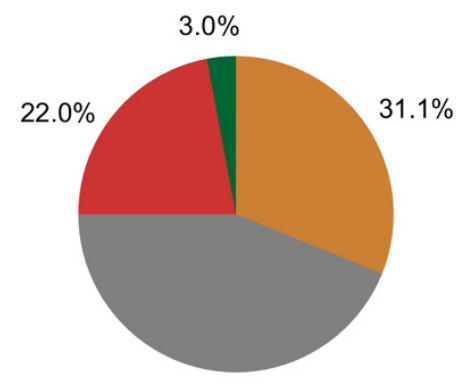

$43.9 \%$

Time segment $11(\mathrm{~N}=440)$

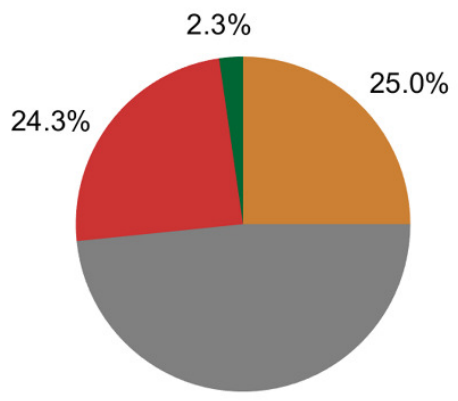

$48.4 \%$

Time segment $3(\mathrm{~N}=740)$

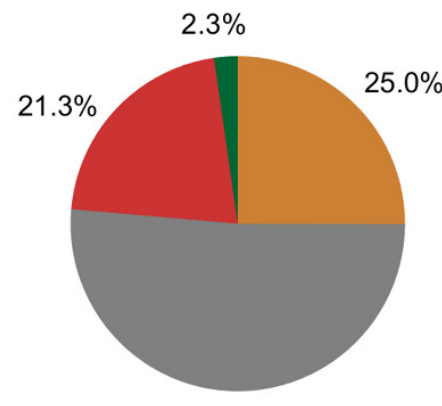

$51.4 \%$

Time segment $6(\mathrm{~N}=703)$

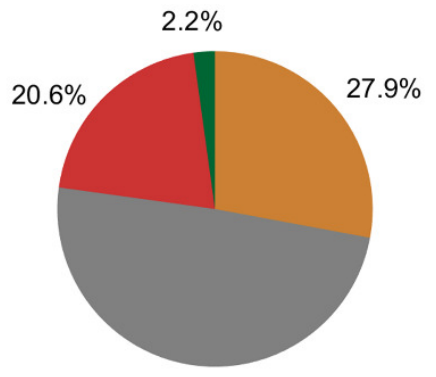

$49.3 \%$

Time segment $9(\mathrm{~N}=548)$

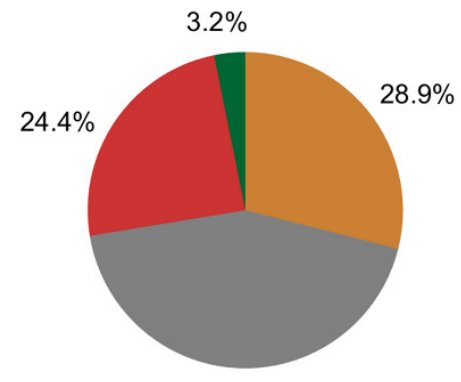

$43.5 \%$

Time segment 12 ( $\mathrm{N}=598)$ 


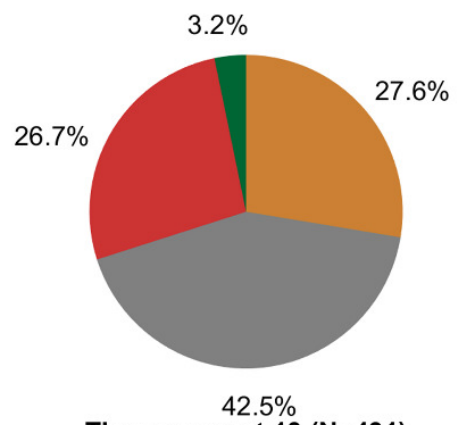

Time segment $13(\mathrm{~N}=431)$

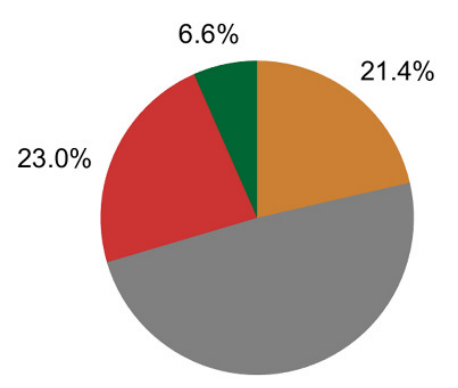

$49.0 \%$

Time segment $16(\mathrm{~N}=514)$

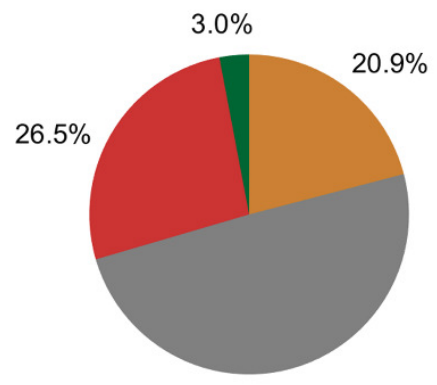

$49.5 \%$

Time segment $19(\mathrm{~N}=535)$

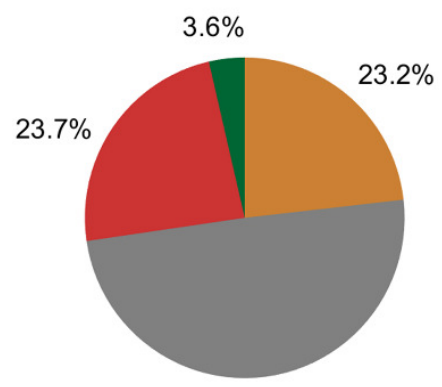

$49.5 \%$

Time segment 22 ( $N=388)$

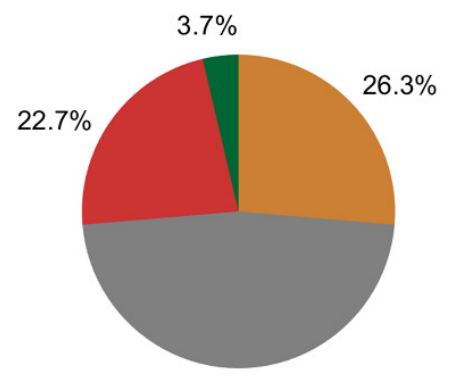

$47.3 \%$

Time segment 14 ( $N=353)$

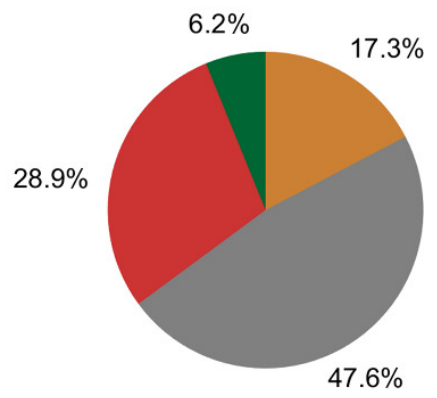

Time segment $17(\mathrm{~N}=681)$

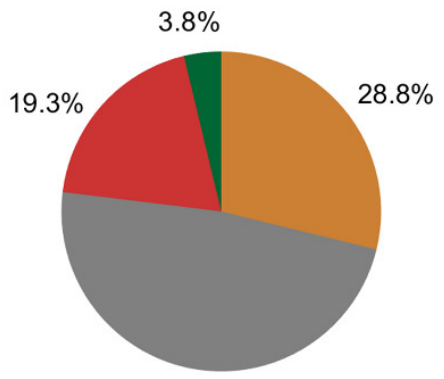

$48.1 \%$

Time segment 20 ( $N=399)$

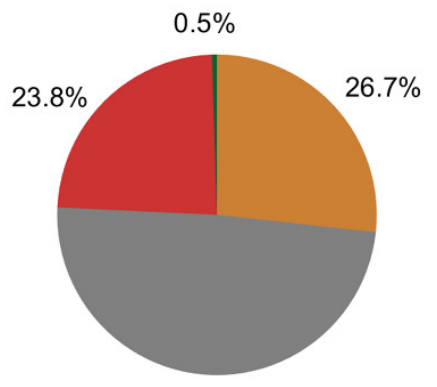

$49.0 \%$

Time segment $23(\mathrm{~N}=404)$
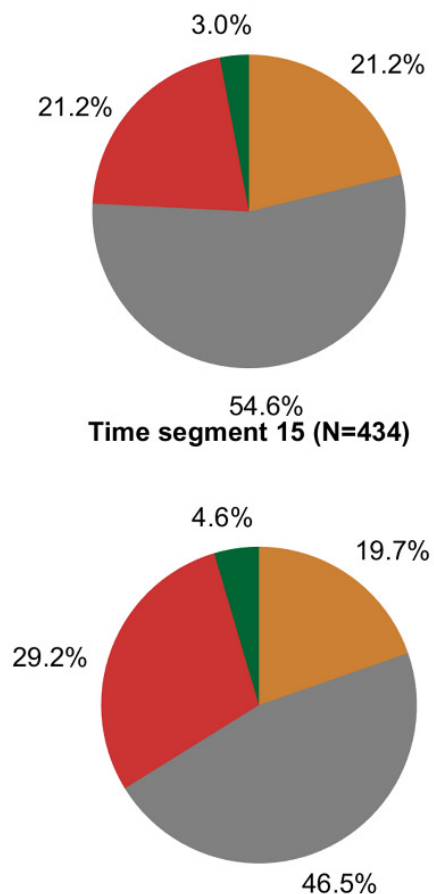

Time segment $18(\mathrm{~N}=742)$

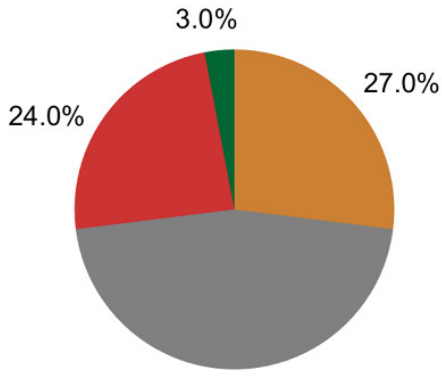

$46.0 \%$
Time segment $21(\mathrm{~N}=\mathbf{3 6 7})$

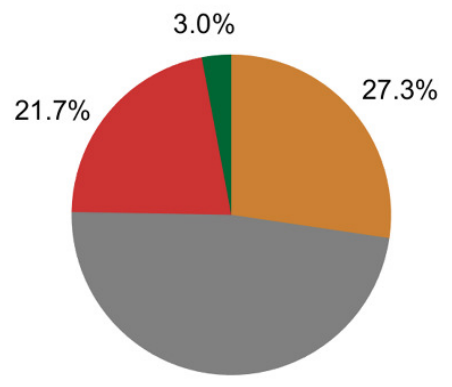

$48.0 \%$

Time segment $24(\mathrm{~N}=538)$ 


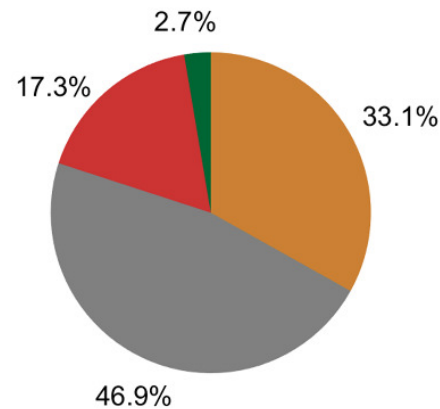

Time segment $25(\mathrm{~N}=450)$

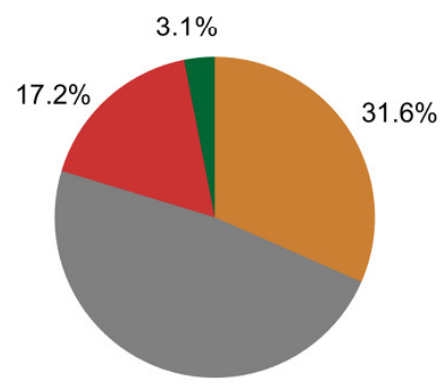

$48.1 \%$

Time segment $28(\mathrm{~N}=551)$

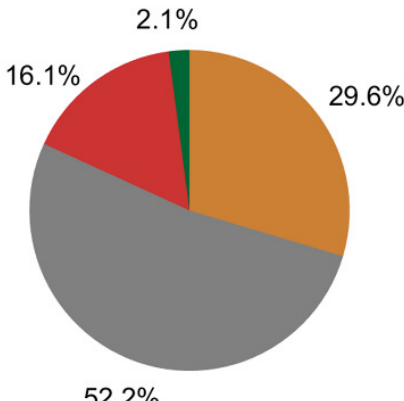

$52.2 \%$

Time segment $26(\mathrm{~N}=429)$

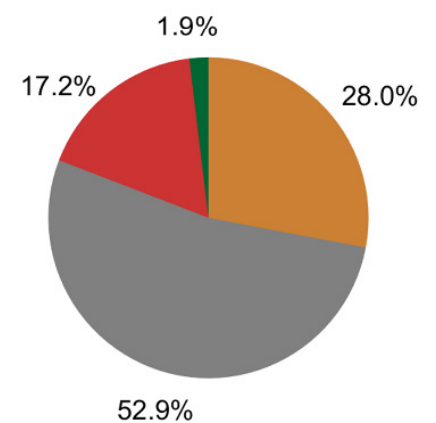

Time segment $29(\mathrm{~N}=522)$

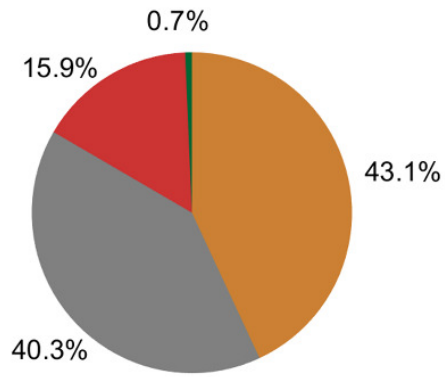

Time segment 27 ( $\mathrm{N}=295)$

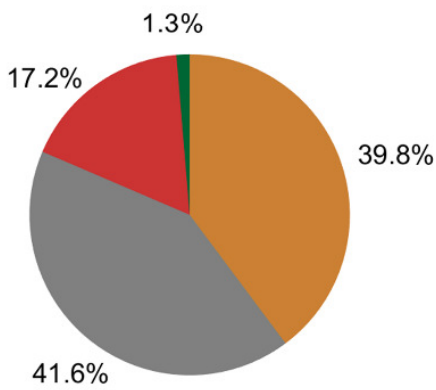

Time segment $30(\mathrm{~N}=377)$

$\begin{array}{llllll}\mathrm{C}_{1} & \mathrm{C}_{2} & \mathrm{C}_{3} & \mathrm{C}_{4}\end{array}$

Figure S2. Temporal variation in $\mathbf{C O}_{2} \mathbf{R R}$ selectivity. Pie charts showing for all 30 time segments the selectivities (\%) of $\mathrm{C}_{1}-\mathrm{C}_{4}$ formation determined for the full ensemble of Ag NPs in $\mathrm{CO}_{2} \mathrm{RR}$ under plasmonic excitation. The number of spectra resulting in each pie chart is listed as $\mathrm{N}$. For this analysis, 68,000 SERS spectra acquired from $61 \mathrm{Ag}$ NP scatterers were analyzed. Of this, 42,000 spectra obtained from $40 \mathrm{Ag}$ NP scatterers are from source data acquired in a previous study. ${ }^{\mathrm{S} 1}$ 


\section{Supporting References}

S1. Devasia, D.; Wilson, A. J.; Heo, J.; Mohan, V.; Jain, P. K. A Rich Catalog of C-C Bonded Species Formed in $\mathrm{CO}_{2}$ Reduction on a Plasmonic Photocatalyst. Nat. Commun. 2021, 12 (1), 2612. 